05

\title{
Структурные превращения на начальных стадиях фрагментации пластически деформируемых поликристаллов. Компьютерный эксперимент
}

\author{
(C) В.В. Рыбин, ${ }^{1,2}$ В.Н. Перевезенцев, ${ }^{1}$ Ю.В. Свирина ${ }^{1}$ \\ ${ }^{1}$ Институт проблем машиностроения РАН, \\ 603024 Нижний Новгород, Россия \\ ${ }^{2}$ Санкт-Петербургский политехнический университет Петра Великого, \\ 195251 Санкт-Петербург, Россия \\ e-mail: rybin.spb@gmail.com
}

(Поступило в Редакцию 8 сентября 2009 г.)

Представлены результаты компьютерного эксперимента по изучению одновременно на микро-, мезо- и макроуровнях рассмотрения эволюции дислокационной структуры в крупной, примыкающей к одному из стыков, области зерна после его макропластической деформации с постоянной скоростью до величины, соответствующей началу стадии развитой пластической деформации. Проанализирован характер распределения плотности дислокаций и дислокационного заряда, величины и степени неоднородности локальной пластической деформации. Установлено, вследствие каких дислокационных перестроек на стыках и изломах большеугловых границ зерен формируются первые оборванные дислокационные границы - мезодефекты, запускающие процесс фрагментации.

DOI: 10.21883/JTF.2017.05.44446.2029

\section{Введение}

Согласно [1-5], фрагментация пластически деформируемых поликристаллов является следствием зарождения и последующей эволюции стыковых и частичных дисклинаций деформационного происхождения (ДП) специфических линейных макро- и линейно-планарных мезо-дефектов ротационного типа [6-14]. Последовательность и иерархическая соподчиненность происходящих при этом процессов ${ }^{1}$ представляются следующей. Внешняя нагрузка создает однородное и одинаковое для всех зерен поле упругих напряжений. В каждом $(i$-м $)$ зерне это поле активирует набор актуальных систем скольжения $(n, b)_{i}$, а однородный поток соответствующих решеточных дислокаций реализует его пластическую деформацию $\varepsilon_{i}^{p l}$ на микроуровне.

В классической дислокационной модели Тэйлора предполагается, что приведенные к единой системе координат тензоры $\varepsilon_{i}^{p l}$ одинаковы для всех зерен поликристалла и при этом равны тензору пластической деформации макрообразца ${ }^{2} \varepsilon^{p l}[15]$ :

$$
\varepsilon_{i}^{p l}=\varepsilon^{p l}, \quad i=1,2,3 \ldots
$$

Формально условию (1) можно удовлетворить, если для каждого $i$-го зерна подобрать свой индивидуальный набор одновременно действующих ${ }^{3}$ актуальных для него систем скольжения. Это допущение лежит в основе

\footnotetext{
${ }^{1}$ Макро-уровень рассмотрения зеренной структуры, мезо-уровень рассмотрения внутризеренной разориентированной структуры, микроуровень рассмотрения однородно ориентированных участков кристалла (расположенных внутри зерна или внутри фрагмента).

2 В том месте, где находится пробное зерно.

${ }^{3}$ В общем случае пяти.
}

современной физической теории пластической деформации поликристаллов и, в частности, позволяет описывать развитие кристаллографических текстур деформации [15]. В рамках его, однако, не представляется возможным даже качественно предсказать явление фрагментации - не менее важное и фундаментальное для физики развитой пластической деформации (РПД) [1]. В классической модели Тэйлора фрагментация поликристаллов в ходе РПД просто-напросто отсутствует. Причина очевидна: принятое в модели Тейлора условие (1) лишь в первом приближении описывает распределение тензоров $\varepsilon_{i}^{p l}$ в пластически деформируемом, но сохраняющем сплошность поликристалле. На самом же деле даже при однородно распределенных в поликристалле напряжениях однородные пластические деформации $\varepsilon_{i}^{p l}$ соседних разориентированных зерен всегда разнятся. Это связано с тремя обстоятельствами. Во-первых, с наличием имманентно присущей всем кристаллическим твердым телам анизотропии их упругих и пластических свойств. Во-вторых, с отличием от нуля вклада упругой составляющей $\varepsilon_{i}^{e l}$ в тензор полной деформации $\varepsilon^{\text {total }}$ каждого $i$-го зерна. В-третьих, с тем, что условие сохранения сплошности поликристаллического агрегата следует относить не к пластической, как это принято в модели Тэйлора, а к полной деформации соседних зерен, [16]:

$$
\varepsilon_{i}^{\text {total }} \equiv \varepsilon_{i}^{p l}+\varepsilon_{i}^{e l}=\varepsilon^{p l}+\varepsilon^{e l} \equiv \varepsilon^{\text {total }} .
$$

Это на первый взгляд незначительное уточнение позволяет серьезно продвинуться в понимании природы фрагментации и ответить на коренной вопрос физики развитой пластической деформации: „почему и как в 
ходе РПД, которая на микроуровне всегда реализуется посредством элементарных трансляционных сдвигов, при $\varepsilon^{p l}>0.2$ с неотвратимостью закона природы появляются ротационные моды пластичности и первоначально однородно ориентированные зерна разбиваются на множество разориентированных фрагментов?“ [17-24].

Цепочка рассуждений, приводящих к ответу на этот вопрос, такова: 1) вопреки предположению (1) на границах зерен пластически деформируемых поликристаллов всегда возникают отличные от нуля скачки пластической деформации $\Delta \varepsilon_{i}^{p l}$, которые при этом всегда уравновешены скачками упругой деформации:

$$
\Delta \varepsilon_{i}^{p l}=-\Delta \varepsilon_{i}^{e l} \neq 0
$$

2) неравные нулю скачки пластической деформации порождают локализованные вдоль линий $j$-стыков границ зерен специфические линейные макродефекты ротационного типа, названные в [1,6-8] „стыковыми дисклинациями деформационного происхождения (СД ДП)“, 3) мощность (величина вектора ротации) СД ДП невелика $\omega^{j} \sim \Delta \varepsilon^{p l} \sim 0.01$, однако монотонно нарастает c ростом макропластической деформации $\varepsilon^{\mathrm{ext}}$ и coответственно с ростом деформирующего макронапряжения $\left.\sigma^{\mathrm{ext}} ; 4\right)$ упругое поле СД ДП $\sigma^{j}(r)$ нарушает ламинарное течение решеточных дислокаций - тем больше, чем больше $\omega^{j}$, в результате чего вблизи СД ДП формируется неоднородное распределение дислокаций; 5) при $\varepsilon^{p l}=\varepsilon_{0}$ эта неоднородность становится столь значительной, что каким-то неизвестным пока образом (одномоментно или постепенно?) трансформируется в специфический планарно-линейный мезодефект ротационного типа — оборванную дислокационную границу деформационного происхождения (ОДГ ДП), [25]; морфологически это событие выглядит так, будто на изломах и стыках большеугловых границ зерен зарождается ОДГ ДП, типичная длина которой обычно составляет несколько микрон, а разориентировка $\Theta_{j} \sim\left(1^{\circ}-3^{\circ}\right)[1]$; 6) повторяясь на разных стыках и изломах границ зерен, зарождение подобных мезодефектов ротационного типа формирует в приграничной зоне область фрагментированной структуры - объемную сетку границ ДП с разориентировками, превышающими $\Theta_{j}$.

В том, что касается общей феноменологии развития дефектной структуры, изложенная модель прекрасно согласуется с имеющимися экспериментальными данными. Однако центральная ее часть (п. 5), относящаяся к механизму зарождения ОДГ ДП, до сих пор экспериментально не верифицирована. Остается непонятным, как именно происходит это структурное превращение и какова его кинетика. Причина очевидна - отсутствие доступных методов изучения столь сложных, многоуровневых, сильновзаимодействующих и иерархически соподчиненных систем. По-видимому, единственным способом разобраться в том, как именно в окрестности стыковой дисклинации в потоке решеточных дислокаций зарождается ОДГ ДП, является компьютерное моделирование [26-31].
Настоящая работа посвящена анализу особенностей постановки компьютерного эксперимента по изучению структурных превращений и процессов зарождения ОДГ ДП на стыковой дисклинации в контролируемых условиях деформации.

\section{1. Особенности методики и постановка задачи}

\section{1. Схема компьютерного эксперимента}

Рассматривается эволюция ансамбля, состоящего в равных частях из положительных $\rho_{+}\left(\varepsilon^{p l}\right)$ и отрицательных $\rho_{-}\left(\varepsilon^{p l}\right)$ решеточных дислокаций, осуществляющих под воздействием внешних нагрузок пластическую деформацию зерна и при этом взаимодействующих между собой, а также с упругим полем стыковой дисклинации ДП мощности $\omega_{j}^{p l}$. Анализируются генерация и аннигиляция дислокаций противоположного знака, а также изменение конфигурации расположения индивидуальных дислокаций относительно друг друга.

Задача рассматривается в простейшей 2D-постановке. В качестве пробного выбирается участок зерна $G 1$ прямоугольной формы высотой $H$ и длиной $L$. Внешняя нагрузка $\sigma^{\text {ext }}$ выбрана такой, что активирует скольжение только в первом зерне. При этом решеточные дислокации не выходят на границы с нормалями $N_{1}, N_{2}, N_{3}$ и тем самым не создают на них дополнительных источников внутренних напряжений.

\section{2. Силы, действующие на дислокации}

Пробная, $i$-я, дислокация, расположенная в точке $\mathbf{r}_{i}$ пластически деформируемого кристалла, находится под воздействием упругих напряжений разной физической природы: внешнего напряжения $\sigma^{\mathrm{ext}}$, напряжения $\sigma^{j}\left(\mathbf{r}_{i}\right)$, создаваемого СД ДП, напряжения $\sigma^{d}\left(\mathbf{r}_{k}-\mathbf{r}_{i}\right)$, создаваемого другими решеточными дислокациями, расположенными в точках с координатами $\mathbf{r}_{k}$. Соответственно сила $f_{G 1}$, действующая на пробную дислокацию в плоскости скольжения зерна $G 1$, является суммой трех составляющих:

$$
f_{G 1}\left(\mathbf{r}_{i}\right)=n_{1}\left[\sigma^{\mathrm{ext}}+\sigma^{j}\left(\mathbf{r}_{i}\right)+\sum_{k} \sigma_{d}\left(\mathbf{r}_{k}-\mathbf{r}_{j}\right)\right] b_{1} .
$$

Первая составляющая

$$
f_{G 1}^{\mathrm{ext}}\left(\mathbf{r}_{i}\right)=n_{1} \sigma^{\mathrm{ext}} b_{1}=b \tau
$$

равна силе, действующей на пробную дислокацию со стороны упругого поля, создаваемого в пробном кристалле внешними нагружающими устройствами; $\tau$ сдвиговая компонента этого поля в плоскости скольжения. Вторая составляющая равна силе, действующей на пробную дислокацию со стороны СД ДП, она выражается как

$$
f_{G 1}^{j}\left(\mathbf{r}_{i}\right)=n_{1} \sigma^{j}\left(\mathbf{r}_{i}\right) b_{1}=\frac{G \omega^{j}}{4 \pi(1-v)} b \sin (2 \varphi),
$$

где $\varphi_{i}$ - полярный угол радиуса-вектора $\mathbf{r}_{i}$. 
Третья составляющая

$$
f_{G 1}^{c o n f}\left(\mathbf{r}_{i}\right)=\sum_{k} n_{i} \sigma^{d}\left(\mathbf{r}_{k}-\mathbf{r}_{i}\right) b_{1}
$$

является „конфигурационной“ силой в том смысле, что зависит только от конкретной конфигурации дислокационного ансамбля. Здесь $k \geq 1, i \leq\left(\rho^{+}+\right.$ $\left.+\rho^{-}\right) L H=\left(N^{+}+N^{-}\right)=N$.

Расчеты конфигурации и характеристик дислокационного ансамбля в окрестности стыковой дисклинации в ходе пластической деформации $\varepsilon^{p l}$ (или при заданной скорости деформации, в момент времени $t$ ), производимой с постоянной скоростью $\dot{\varepsilon}^{p l}$, являются главной задачей настоящего компьютерного моделирования.

В настоящей работе она решается методом итераций.

\section{3. Итерационная процедура}

Процесс пластической деформации пробного кристалла рассматривается как непрерывная цепь $M$ малых приращений деформаций $\Delta \varepsilon_{m}^{p l}$ :

$$
\varepsilon^{p l} \equiv \varepsilon_{M}^{p l}=\sum_{1}^{M} \Delta \varepsilon_{m}^{p l}=\sum_{1}^{M}\left(\varepsilon_{m}^{p l}-\varepsilon_{m-1}^{p l}\right),
$$

где $\varepsilon_{m}^{p l}$ - суммарная пластическая деформация пробного кристалла к моменту $t_{m}$ окончания $m$-й итерации. Скорость пластической деформации $\dot{\varepsilon} p l$ считается постоянной на каждой итерации. Величина парциальной деформации $\Delta \varepsilon_{m}^{p l}$ выбирается из соображений удобства вычислений и обычно определяется текущей плотностью дислокаций. В настоящей работе в среднем $\Delta \varepsilon_{m}^{p l}$ составляла $2 \cdot 10^{-4}$.

Предполагается, что вначале каждой $m$-й итерации по результатам предыдущей, $(m-1)-$ й, задается исходная дислокационная конфигурация, состоящая из $N_{m-1}^{+}$положительных и $N_{m-1}^{-}$отрицательных дислокаций. Для каждой $i$-й дислокации $1 \leq i \leq N_{m-1} \equiv N_{m-1}^{+}+N_{m-1}^{-}$по формулам (4)-(6) вычисляются действующие на нее мгновенные силы $f_{G 1, m}\left(r_{i}\right)$ и $f_{G 1, m}^{c o n f}\left(\mathbf{r}_{i}\right)$. Зная эти силы, в рамках той или иной физической модели можно вычислить мгновенную скорость $V_{m}\left(\mathbf{r}_{i}\right)$ для каждой из дислокаций, образующих исходную для $m$-й итерации конфигурацию. В настоящей работе принята модель квазивязкого движения дислокации, т. е. предполагается, что температура и внешнее напряжение обеспечивают надбарьерное скольжение дислокаций. Такая модель наилучшим образом подходит для описания процесса фрагментации. В ней скорость дислокации оказывается пропорциональной силе $f\left(\mathbf{r}_{i}\right)$ :

$$
V_{m}\left(\mathbf{r}_{i}\right)=H_{h}\left(\sigma_{c}\right) B(T) f\left(\mathbf{r}_{i}\right),
$$

где $B(T)$ - зависящий от температуры коэффициент подвижности, $H_{h}\left(\sigma_{c}\right)-$ ступенчатая функция Хэвисайда, равная нулю при $\sigma \leq \sigma_{c}$ и единице при обратном неравенстве.
По найденной скорости дислокаций и принятой длительности итерации

$$
\Delta t_{m}=\Delta \varepsilon_{m}^{p l} / \dot{\varepsilon}^{p l}
$$

вычисляем пробеги $\lambda_{m}\left(\mathbf{r}_{i}\right)$ для всех наличествующих в кристалле дислокаций. Таким образом, взяв конфигурацию дислокаций, конечную для предыдущей $(m-1)$-й итерации, мы в процессе $m$-й итерации находим новую конфигурацию, которая, в свою очередь, будет исходной для следующей $(m+1)$-й итерации.

\section{4. Кинетика изменения плотности дислокаций}

Эволюция дислокационного ансамбля в ходе $m$-й итерации включает в себя не только изменение его исходной конфигурации при сохранении плотности $\rho_{m}\left(\varepsilon_{m}^{p l}\right)$ образующих его дислокаций, но и изменение самой этой плотности. Для этого учитываются процессы аннигиляции и размножения дислокаций.

1.4.1. Аннигиляция дислокаций. Аннигиляция двух дислокаций противоположного знака происходит, если они, скользя в одной и той же плоскости в противоположных направлениях, сближаются на критическое расстояние $x_{c}$. В первом приближении $x_{c}$ можно определить как расстояние, на котором сила притяжения двух дислокаций противоположного знака равняется или превышает силу (5), откуда

$$
x_{c} \approx \frac{b}{2 \pi(1-v)}\left(\frac{G}{\sigma^{\mathrm{ext}}}\right) .
$$

Аннигиляция дислокаций, движущихся навстречу друг другу в разных параллельных плоскостях, происходит с сечением $S_{a}=\pi x_{a}^{2} / 4$, где $x_{a}=0.25 x_{c}$

1.4.2. Размножение дислокаций. В реальных трехмерных кристаллах роль источников новых дислокаций играют сегменты дислокационной сетки. Под действием упругих напряжений они „раздуваются“ в замкнутые дислокационные петли по механизму ФранкаРида. Возникновение подобных петель можно рассматривать как стохастический, однородный по объему процесс генерации новых дислокаций. Образование новых дислокационных сегментов может происходить и по механизму двойного поперечного скольжения винтовых компонент дислокационных петель. Этот процесс также имеет вероятностный характер и также однородно охватывает объем пластически деформируемого кристалла [32].

В двумерном кристалле осуществить подобные механизмы размножения дислокаций не представляется возможным. Чтобы скорректировать эту чисто геометрическую специфику, сохраняя при этом физическую суть процесса пластической деформации, Ван дер Гиссен предложил моделировать размножение дислокаций в 
$2 D$-постановке чисто формальным способом - введя случайную по времени и однородную по объему генерацию пар дислокаций противоположного знака [26].

В настоящей работе мы воспользовались идеей Ван дер Гиссена, откорректировав ее в части однородности генерации дислокационных пар по объему пластически деформируемого кристалла. А именно ввели запрет на зарождение дислокационных пар в точках, расположенных от уже существующих дислокаций на расстоянии, меньшем, чем критическая высота выброса при двойном поперечном скольжении винтовых дислокаций. Учет этого эффекта приводит к замедлению генерации новых дислокаций в местах скопления уже существующих.

\subsection{3. Кинетика изменения средней плотности} дислокаций. Зная для $m$-й итерации усредненные по кристаллу значения исходной плотности дислокаций $\rho_{m}$, пробега дислокаций $\lambda_{m}$ и скорости дислокаций $V_{m}$, можно написать уравнение для скорости пластической деформации кристалла в конечных разностях

$$
\dot{\varepsilon}^{p l}=\dot{\varepsilon}_{m}^{p l}=\rho_{m} b V_{m}+\frac{\left(\rho_{m+1}-\rho_{m}\right)}{\Delta t_{m}} b \lambda_{m} .
$$

Принимая во внимание, что величина $\lambda_{m} / \Delta t_{m}$ примерно равна средней скорости дислокаций на $m$-й итерации, из (10) находим

$$
\rho_{m+1} \approx \frac{\dot{\varepsilon}^{p l}}{b V_{m}} .
$$

Это означает, что до начала $m$-й итерации мы должны внести в пробный кристалл дополнительно

$$
\Delta N_{m}=L H\left(\frac{\dot{\varepsilon}^{p l}}{b V_{m}}-\rho_{m}\right)
$$

новых, хаотически разбросанных по нему дислокаций.

Таким образом, в дополнение к сказанному в разд. 1.3 исходная конфигурация дислокаций на момент начала $m$-й итерации представляет собой суперпозицию упорядоченных в ходе предыдущей итерации дислокаций, дополненных новыми хаотически разбросанными по кристаллу дислокациями в количестве $\Delta N_{m}$ штук.

1.4.4. Краевой эфффект. При компьютерном моделировании дислокационной структуры пластически деформируемого кристалла в приграничных, протяженностью порядка $\lambda_{m}$, областях наблюдается локальное снижение плотности дислокаций. Такой „краевой“ эффект объясняется вынужденной нескомпенсированностью дислокационных потоков на границах пробного кристалла. Ошибок, обусловленных наличием „краевого“ эффекта, легко избежать, увеличив размеры пробного кристалла и (или) анализируя дислокационную структуру в областях, удаленных от его границ на расстояние, превышающее $\lambda_{m}$.

\section{2. Параметры структуры ДП, подлежащие изучению методами компьютерного моделирования}

Для характеристики пространственной однородности и неоднородности развития дислокационных структур в объеме пробного кристалла, в том числе вблизи стыковой дисклинации ДП, в нем выделяли шесть зон $(a, b, c, d, e, f)$ размерами $5 \times 10 \mu \mathrm{m}^{2}$ каждая. Размеры пробного кристалла при этом составляют $5 \times 60 \mu \mathrm{m}^{2}$.

Для пробного кристалла в целом и для каждой зоны в отдельности качественно и количественно характеризовали морфологические особенности дислокационной структуры, сформировавшейся при пластической деформации с постоянной скоростью $\dot{\varepsilon}^{p l}$ до разных значений $\varepsilon^{p l}$, в том числе при отсутствии СД ДП и при наличии ее (мощностью $\omega^{j}=0.01$ и $0.04 \mathrm{rad}$ ).

Количественный анализ дислокационных структур ДП, сформировавшихся в пробном кристалле, а также в различных его зонах, включал в себя определение:

1) плотности дислокаций $\rho(\varepsilon)$,

2) плотности дислокационного заряда $\rho_{+}^{c h}(\varepsilon), \rho_{-}^{c h}(\varepsilon)$,

$3)$ средней скорости $V(\varepsilon)$ и среднего пробега дислокаций $\lambda(\varepsilon)$,

4) средней величины $\varepsilon$ и средней скорости пластической деформации $\dot{\varepsilon}_{i}(\varepsilon)$. Отдельно анализировали изменение дислокационной структуры, сформировавшейся вблизи СД ДП, после разгрузки пробного кристалла и последующей выдержки его при температуре, равной температуре деформации (статический возврат).

Согласовывали с экспериментальными данными только средние для пробного кристалла значения $\varepsilon^{p l}, \dot{\varepsilon}^{p l}$, $\rho$ и $\rho^{c h}$. Что же касается отдельных зон пробного кристалла, то для них характер изменения перечисленных параметров с ростом $\varepsilon^{p l}$ неизвестен и мог быть выявлен только в ходе настоящего компьютерного эксперимента.

\section{3. Общая постановка компьютерного эксперимента}

Согласно существующим представлениям, СД ДП формируются уже на самых ранних стадиях упругопластического течения поликристалла. Вначале их мощность пренебрежимо мала, однако монотонно нарастает по мере увеличения $\varepsilon^{p l}$. Кинетика этого процесса в настоящее время неизвестна. Между тем экспериментальные наблюдения показывают, что первые ОДГ ДП появляются в поликристалле при $\varepsilon^{p l} \approx 0.2$ и имеют разориентировку, близкую к (0.02-0.04) rad. Согласно существующим модельным представлениям [4,9], это означает, что мощность $\omega^{j}$ породивших их СД ДП примерно вдвое больше.

Чтобы разобраться, почему и как происходит зарождение элементарной СД ДП, необходимо промоделировать эволюцию дислокационной структуры в интервале деформаций $0<\varepsilon^{p l} \leq 0.2$. Причем, очевидно, 


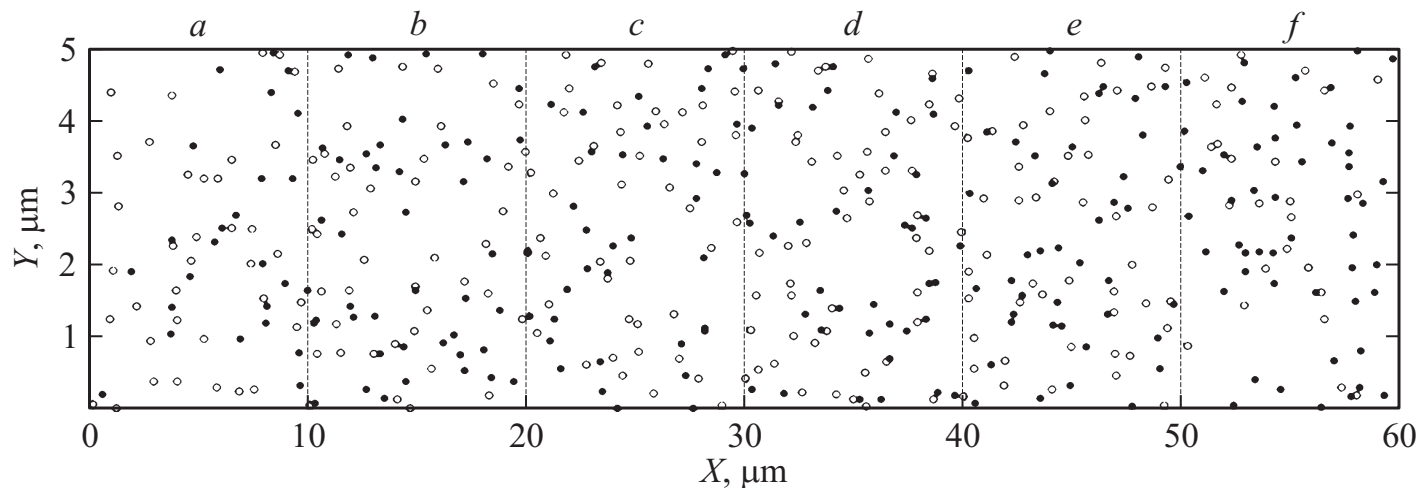

$a$
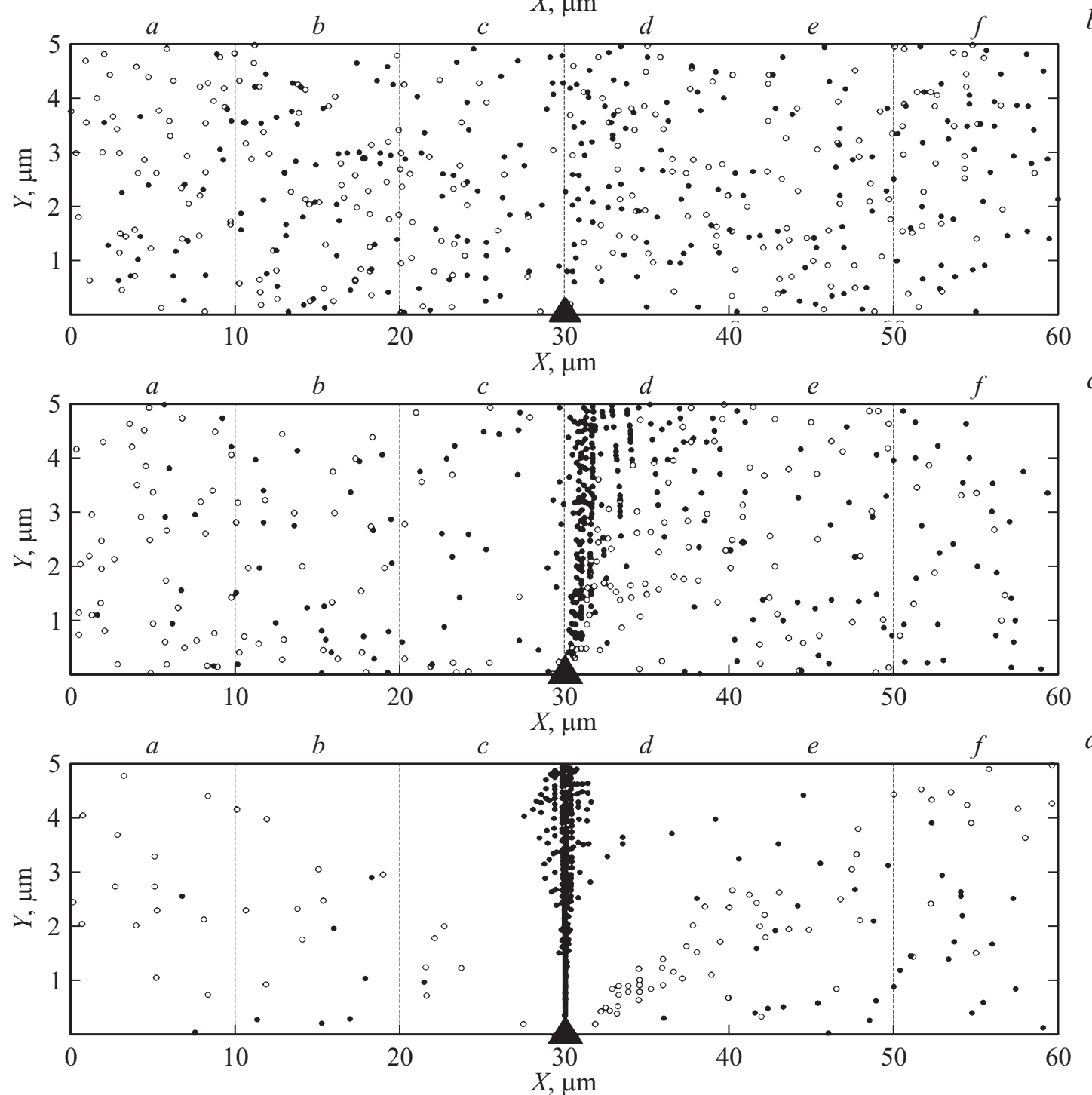

Рис. 1. Дислокационная структура, формирующаяся в процессе пластической деформации пробного кристалла, $\omega^{j}: a-0, b-$ $0.01, c-0.04$ и после разгрузки $-d$. Пустые и черные кружочки - соответственно отрицательные и положительные дислокации.

что эволюцию эту необходимо рассматривать с учетом неразрывности и соподчиненности процессов, происходящих на макро-, мезо- и микроуровнях. Непрерывное увеличение $\varepsilon^{p l}$ сопровождается непрерывным увеличением $\omega^{j}$. Это, в свою очередь, вызывает непрерывное нарастание дисклинационной составляющей в поле сил, действующих на пробную решеточную дислокацию. При некоторой $\varepsilon^{p l}=\varepsilon_{0}$ непрерывно нарастающая мощность стыковой дисклинации достигнет такой величины $\omega^{j}\left(\varepsilon_{0}\right)$, что в потоке проходящих мимо нее решеточных дислокаций произойдет зарождение оборванной дислокационной границы ДП. 

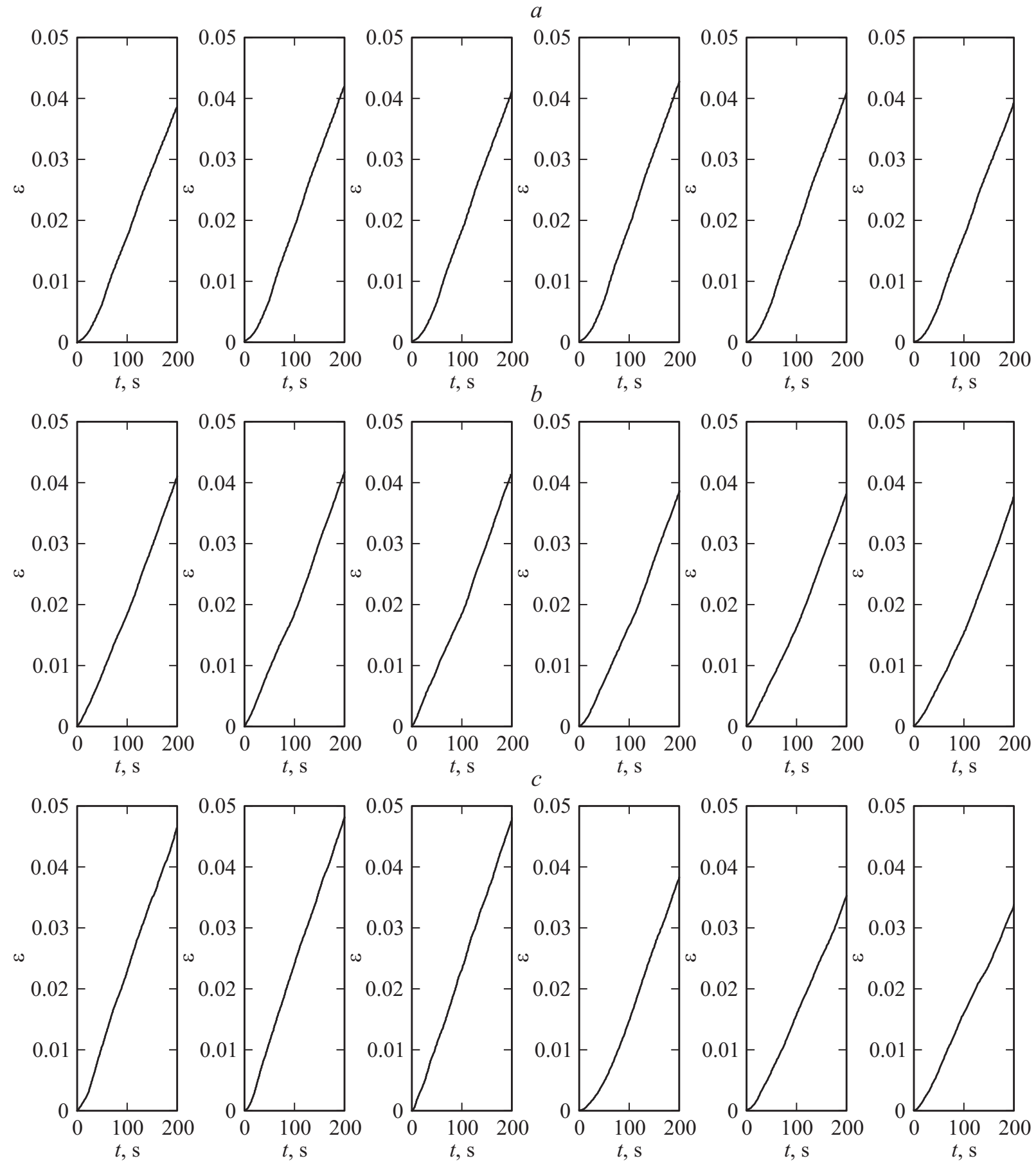

Рис. 2. Временны́е зависимости величин деформации для зон $(a, b, c, d, e, f)$ пробного кристалла, $\omega^{j}: a-0, b-0.01, c-0.04$.

Ставить задачу о компьютерном моделировании процесса зарождения ОДГ ДП в столь общей постановке пока не представляется возможным, поскольку в настоящее время отсутствует физическая модель, позволяющая рассчитывать мощность $\omega^{j}$ как функцию величины $\varepsilon^{p l}$. В настоящей работе моделировали эволюцию дислокационной структуры пробного кристалла для трех конкретных значений мощности стыковой дисклинации ДП $\left(\omega^{j}=0, \omega^{j}=0.01\right.$ и $\left.0.04 \mathrm{rad}\right)$. В шкале истинных логарифмических деформаций макрообразца это соответствует значениям $\varepsilon^{p l}$, примерно равным 0 , 0.01 и 0.04. Макроскопическая скорость пластической деформации $\dot{\varepsilon}^{p l}$ во всех трех случаях принималась равной $2 \cdot 10^{-4} \mathrm{~s}^{-1}$.

Во всех компьютерных экспериментах в исходном состоянии пробный кристалл не содержал дислокаций. По мере увеличения числа итерационных процедур плотность дислокаций в нем монотонно нарастала и постепенно приближалась к величине порядка $2 \cdot 10^{12} \mathrm{~m}^{-2}$. Дислокационные структуры ДП, эволюционируя, при- 

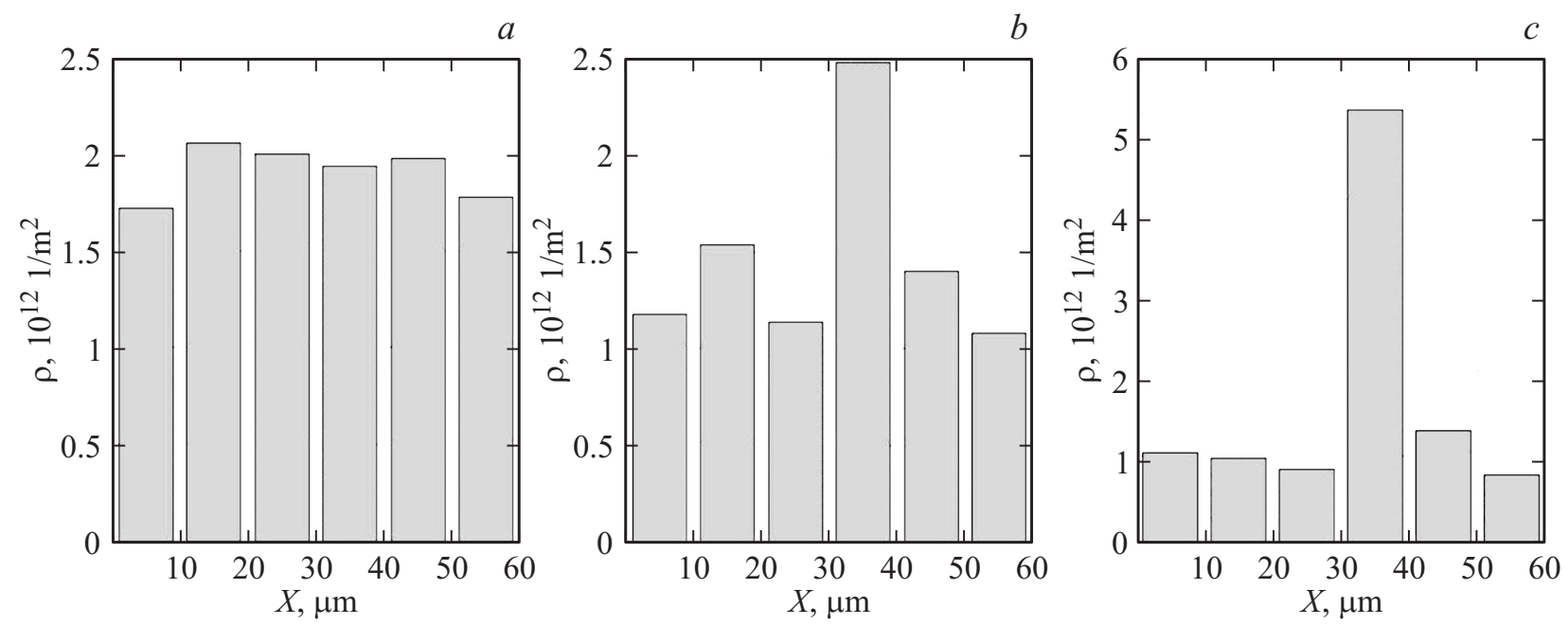

Рис. 3. Распределение плотности дислокаций $\rho$ по зонам $(a, b, c, d, e, f)$ пробного кристалла, $\omega^{j}: a-0, b-0.01, c-0.04$.

ближались к некоторому устойчивому состоянию. Полученные в ходе компьютерного эксперимента характеристики таких морфологически устойчивых структурных состояний рассматриваются ниже.

\section{4. Результаты компьютерного эксперимента}

\section{1. Мощность стыковой дисклинации $\omega^{j}=0$}

Дислокационная структура, сформировавшаяся в пробном кристалле, продеформированном до величины деформации $\varepsilon^{p l}=0.04$ со скоростью $\dot{\varepsilon}^{p l}=2 \cdot 10^{-4} \mathrm{~s}^{-1}$, представлена на рис. $1 a$. Под воздействием конфигурационных сил движущиеся дислокации перераспределяются таким образом, что на фоне в среднем однородной по кристаллу плотности дислокаций в их ансамбле формируется слабовыраженная ячеистая структура. Характерный размер ячейки составляет $0.5 \mu \mathrm{m}$, а их разориентировки не превышают $10^{-4} \mathrm{rad}$.

Зависимость величины и скорости пластической деформации от времени нагружения с разбивкой по зонам $(a, b, c, d, e, f)$ пробного кристалла представлена на рис. $2, a$. Видно, что на всем протяжении компьютерного эксперимента кристалл в целом, а также и все составляющие его зоны испытывают примерно одинаковую пластическую деформацию с одинаковой постоянной скоростью $\dot{\varepsilon}^{p l}=2 \cdot 10^{-4} \mathrm{~s}^{-1}$. Поскольку длительность эксперимента равнялась $200 \mathrm{~s}$, итоговая деформация $\varepsilon^{p l}$ составила примерно $4 \%$.

Распределения плотности дислокаций $\rho$ внутри пробного кристалла после такой деформации представлены (с разбивкой по зонам) на рис. 3, $a$. С точностью до статистического разброса распределение дислокаций в центральной части пробного кристалла можно считать однородным с плотностью $\rho \approx 2 \cdot 10^{12} \mathrm{~m}^{-2}$. Исключение составляют две крайние зоны $(a$ и $f)$, где плотность дислокаций снижается примерно на 20\%. Так проявляется краевой эффект, связанный с нескомпенсированностью дислокационных потоков на выходе из пробного кристалла.

Еще более явно краевой эффект сказывается на распределении относительной плотности дислокационного заряда $\rho^{c p} / \rho$ (рис. 4, $a$ ). В зоне $f$ резко, примерно в 6 раз, повышается доля положительных дислокаций, которые под действием внешних напряжений движутся слева направо и выходят из кристалла через его правую границу. В зоне $a$ по аналогичной причине скапливаются отрицательные дислокации. В двух центральных зонах с точностью до статистического разброса имеет место равенство положительных и отрицательных дислокаций. В зонах $b$ и $e$ это равенство нарушено, но незначительно. Отсюда следует, что, строго говоря, при выбранной геометрии кристалла результаты компьютерного моделирования дислокационной структуры в окрестностях стыковой дисклинации оказываются статистически надежными только в пределах зон $c$ и $d$.

Этот критерий заведомо выполняется при анализе механизмов зарождения ОДГ ДП, который, как будет показано, реализуется именно в этих зонах.

\section{2. Мощность стыковой дисклинации $\omega^{j}=0.01$}

Типичная дислокационная структура пробного кристалла, содержащего стыковую дисклинацию мощностью $\omega^{j}=0.01$, после пластической деформации $\varepsilon^{p l}=0.04$ представлена на рис. $1, b$. В среднем по кристаллу плотность дислокаций остается прежней, $\rho \approx 2 \cdot 10^{12} \mathrm{~m}^{-2}$. Однако она перестала быть однородной и этим качественно отличается от рассмотренной выше. Распределение дислокаций по зонам (рис. 3,b) демонстрирует, что, действительно, особенно заметные изменения произошли в зонах, непосредственно примыкающих к стыковой дисклинации. В зоне $d$ плотность дислокаций повысилась до значений $\rho \cong 2.5 \cdot 10^{12} \mathrm{~m}^{-2}$, в 

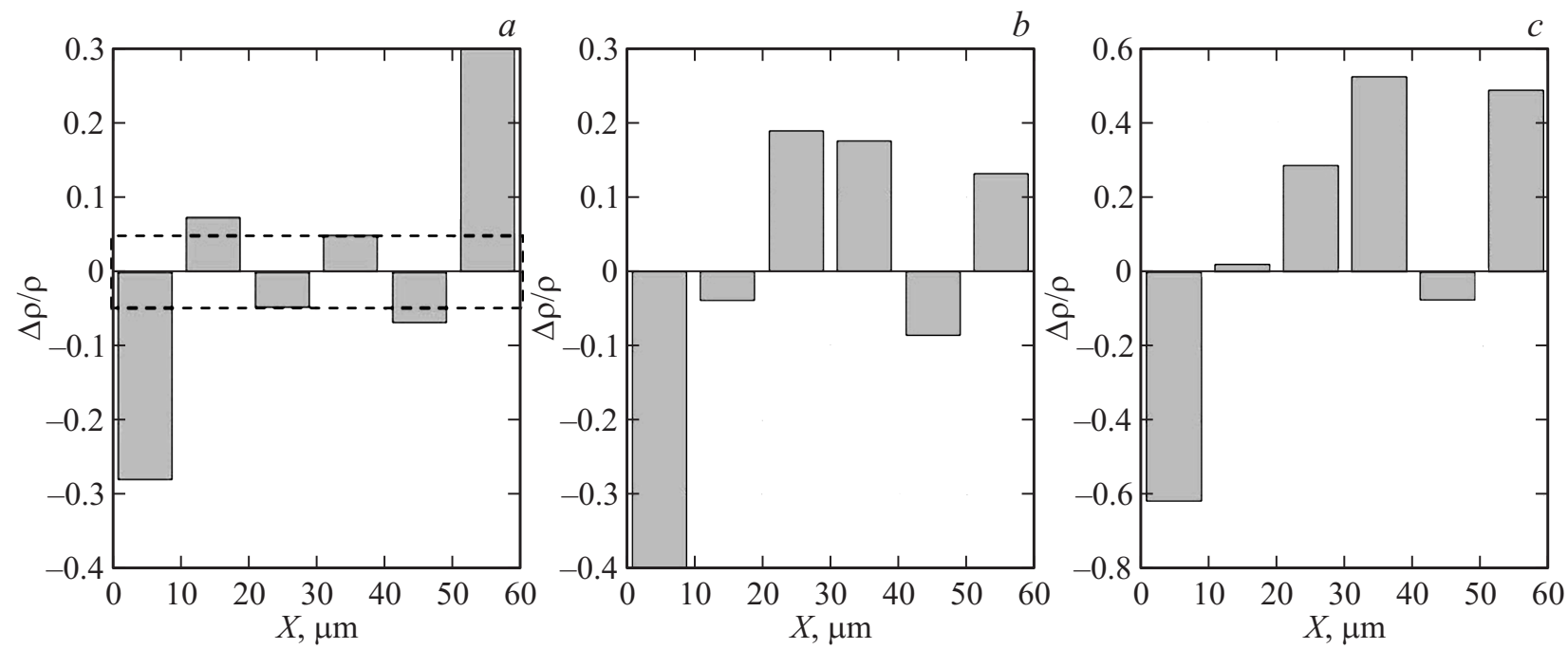

Рис. 4. Распределение относительной плотности дислокационного заряда по зонам $(a, b, c, d, e, f)$ пробного кристалла, $\omega^{j}: a-0$ (штриховой пунктирной линией показана величина статистического отклонения по результатам десяти экспериментов), $b-0.01$, $c-0.04$.

соседней зоне $c$ снизилась до $\rho \cong 1.2 \cdot 10^{12} \mathrm{~m}^{-2}$. Иными словами, при пластической деформации пробного кристалла справа от дисклинации создается динамическое сгущение, а слева - динамическое разряжение дислокации. При этом, как следует из рис. $4, b$, в обоих зонах превалируют положительные дислокации.

\section{3. Мощность стыковой дисклинации $\omega^{j}=0.04$}

При мощности стыковой дисклинации $\omega^{j}=0.04$ вблизи нее начинает формироваться дислокационная граница ДП (рис. $1, c)$. Она имеет сложное слоистое строение и представляет собой систему параллельных дислокационных стенок различной длины, состоящих в основном из положительных дислокаций. Разориентировки на таких „зародышах границ ДП“ чрезвычайно малы и составляют примерно $0.001 \mathrm{rad}$. Слева и справа от дисклинации формируются области соответственно с пониженной и повышенной плотностью дислокаций (рис. $3, c$ ). При этом слева от дисклинации (в зонах $b$ и $c$ ) и справа от нее (в зонах $e$ и $f$ ) наблюдается избыток положительных дислокаций.

Это связано с особенностями упругого поля дисклинации [9]. В зонах, расположенных слева от дисклинации, сила (6), действующая со стороны дисклинации как на положительные, так и на отрицательные дислокации, совпадает по направлению с силой, действующей на них со стороны внешнего напряжения. В противоположность этому в зонах, расположенных справа от нее, силы, действующие на дислокацию со стороны дисклинации и внешней нагрузки, направлены в разные стороны. По этой причине пластическая деформация пробного кристалла справа от дисклинации в среднем идет с меньшей скоростью, чем слева от нее (рис. 2,c). При переходе положительных дислокаций из левой в правую часть пробного кристалла упругое поле дисклинации особенно резко тормозит их в зоне $d$. Именно там происходит накопление избыточной плотности положительных дислокаций. Когда в локальных областях вблизи дисклинации избыточная плотность положительных дислокаций достигает критического значения, конфигурационные силы перестраивают их в энергетически более выгодные конфигурации. Так происходит формирование „зародышей границ ДП“. В ходе продолжающейся деформации число таких зародышей нарастает. Они объединяются между собой и образуют искомую дислокационную границу ДП, зародившуюся на стыковой дисклинации. Однако этот, казалось бы, очевидный вывод необходимо подкрепить более детальными исследованиями. Они будут приведены в следующих публикациях. Оценка же разориентировки представленного на рис. 1, $c$ образования, которое можно интерпретировать как начальную стадию формирования границы ДП дает величину $\Theta \approx 0.017 \mathrm{rad}$.

\section{4. Релаксация дислокационной структуры после разгрузки пробного кристалла}

После снятия внешнего напряжения дислокационная структура, показанная на рис. $1, c$, релаксирует. При этом находившиеся в областях „сгущения“ и „разряжения“ положительные дислокации стекают на зародыш границы ДП, превращая его в устойчивую границу наклона. Она располагается перпендикулярно действующей плоскости скольжения и имеет распушенную вершину (рис. $1, d)$. Разориентировка на этой отрелаксированной границе заметно увеличилась и стала равной $0.02 \mathrm{rad}$, что полностью соответствует теоретическим представлениям [4]. Слева и справа от нее наблюдаются области с относительно высокой плотностью отрицательных дислокаций. 


\section{5. Обсуждение и выводы}

В настоящей работе мы использовали максимально упрощенную модель пластической деформации кристалла: элементарная кристаллография, единственная система скольжения, простейшая схема нагружения, отсутствие переползания и других температурнообусловленных механизмов движения дислокаций. Однако даже при такой идеализации процесса пластической деформации удается объяснить, почему и как в реальных поликристаллах, различающихся кристаллографией, химическим и фазовым составами, деформируемых в чрезвычайно широком диапазоне температурно-скоростных режимов и технологических схем нагружения, при $\varepsilon \geq 0.2$ на стыках и изломах большеугловых границ зерен появляются первые ОДГ ДП с разориентировками $\Theta \approx(0.01-0.03) \mathrm{rad}$. Почему и как с неодолимостью закона природы начинается фрагментация кристалла.

Парадоксально, но именно это предельное упрощение модели придает фундаментальность полученным результатам. Очевидно, оно не затронуло главного того, что на самом деле и является истинной причиной потери ориентационной устойчивости кристаллов на стадии развитой пластической деформации. Причиной, которая незримо остается инвариантной для всего многообразия проявления фрагменации.

Из представленных в настоящей работе результатов следует, что таким инвариантом является стыковая дисклинация деформационного происхождения. Этот необнаруживаемый по морфологическим признакам линейный макродефект ротационного типа образуется при пластической деформации поликристаллического агрегата по чисто геометрическим причинам, вне зависимости от характеристик материала и режима деформации. Мощность СД ДП $\omega^{j}$ монотонно нарастает по мере увеличения $\varepsilon^{p l}$. Пропорционально нарастает интенсивность генерируемого ею поля упругих напряжений. Это приводит к тому, что вблизи стыков зерен, содержащих СД ДП, происходят следующие изменения:

1) пластическая деформация теряет свою однородность и первоначально однородно деформируемый кристалл разбивается на мезообъемы, пластические деформации которых все больше и больше различаются (рис. 2),

2) первоначально однородная плотность дислокаций перестает быть таковой, и в кристалле формируются мезообъемы с повышенной и пониженной плотностью $\rho$ (рис. 3);

3) происходит сепарация дислокаций по знаку. Формируются мезообъемы с преимущественно положительными или отрицательными дислокациями (рис. 4).

Особенно активно эти процессы идут в мезообъемaх, непосредственно примыкающих к стыку (зоны $c$ и $d$ на рис. 1-4). На определенной стадии пластической деформации поликристалла, $\varepsilon^{p l} \approx \varepsilon_{0}$, когда величина $\omega^{j}$ достигнет своего критического значения $\omega_{0}^{j}$, на границе наиболее активных мезообъемов формируется оборванная дислокационная граница с разориентировкой $\Theta \approx 0.5 \omega_{0}^{j}$. Удивительно, что в рамках столь простой модели удается получить значения критических параметров $\omega_{0}^{j}$ и $\Theta_{0}$, хорошо согласующиеся с экспериментальными данными.

Результаты компьютерного эксперимента по детализации микромеханизма и кинетики процесса зарождения на стыковой дисклинации первой ОДГ ДП будут приведены в следующей публикации.

Исследование выполнено за счет гранта Российского научного фонда (проект № 15-13-20030).

\section{Список литературы}

[1] Рыбин В.В. Большие пластические деформации и разрушение металлов. М.: Металлургия, 1986. 224 с.

[2] Рыбин В.В., Вергазов А.Н., Лихачёв В.А. // ФММ. 1974. T. 37. C. $620-624$.

[3] Золоторевский Н.Ю., Рыбин В.В. Фрагментация и текстурообразование при деформации металлических материалов. СПб.: Изд-во Политех. ун-та, 2014. 208 с.

[4] Сарабанов Г.Ф., Перевезенцев В.Н., Рыбин В.В. Основы кинетической теории формирования разориентированных структур при пластической деформации металлов. Н. Новгород: Литера, 2011. 357 с.

[5] Рыбин В.В., Перевезенцев В.Н., Свирина Ю.В. // ЖТФ. 2016. Т. 86. Вып. 5. С. 100-105.

[6] Рыбин В.В., Зисман А.А., Золоторевский Н.Ю. // ФТТ. 1985. Т. 27. Вып. 1. С. 181-186.

[7] Rybin V.V., Zisman A.A., Zolotorevsky N.Yu. // Acta Met. Mater. 1993. Vol. 41. N 7. P. 2211-2217.

[8] Zisman A.A., Rybin V.V. // Acta Mater. 1996. Vol. 44. N 1. P. 403-407.

[9] Romanov A.E., Vladimirov V.I. Disclinations in crystalline solids, in F.R.N. Nabarro ed. „Dislocations in solids“, NorthHolland, Amsterdam, 1992. Vol. 9. P. 191-402.

[10] Romanov A.E. // Europ. J. Mech. Sol. 2003. Vol. 22. P. 727-741.

[11] Romanov A.E., Kolesnikova A.L. // Progr. Mater. Sci. 2009. Vol. 54. P. 740-769.

[12] Romanov A.E., Kolesnikova A.L., Ovid'ko I.A., Aifantis E.C. // Mater. Sci. Eng. A. 2009. Vol. 503. P. 62-67.

[13] Gutkin M.Y., Mikaelyan K.N., Romanov A.E., Klimanek P. // Phys. Stat. Sol. A. 2002. Vol. 193. P. 35.

[14] Gutkin M.Y., Ovid'ko I.A. // Appl. Phys. Lett. 2005. Vol. 87. P. 251916.

[15] Taylor G.I. // J. Inst. Metals. 1938. Vol. 62. P. 307-324.

[16] De Wit R. // J. Phys. C: Sol. Stat. Phys. 1972. Vol. 5. P. 529-534.

[17] Валиев Р.З., Александров И.В. Объемные наноструктурные металлические материалы. М.: Академкнига, 2007. $398 \mathrm{c}$.

[18] Zehetbauer M.J., Valiev R.Z. Nanomaterials by Severe Plastic Deformation. Germany: Wiel_VCH, Weinheim, 2004. 850 p.

[19] Langdon T.G. // Acta Mater. 2013. Vol. 61. P. 7035-7059.

[20] Estrin Y., Vinogradov A. // Acta Mater. 2013. Vol. 61. P. $782-817$.

[21] Mulyukov R.R., Imayev R.M., Nazarov A.A. // J. Mater. Sci. 2008. Vol. 43. N 23-24. P. 257-7263. 
[22] Колобов Ю.Р., Грабовецкая Г.П., Дударев Е.Ф., Иванов К.В. // Вопросы материаловедения. 2004. № 1. C. $56-63$.

[23] Salishchev G., Zherebtsov S., Mironov S., Belyakov A. // Mater. Characterization. 2010. Vol. 61. N 7. P. 732-739.

[24] Pilyugin V.P., Voronova L.M., Gapontseva T.M., Chashchukhina T.I., Degtyarev M.V. // Intern. J. Refract. Metal. Hard Mater. 2014. Vol. 43. P. 59-63.

[25] Вергазов А.Н., Лихачёв В.А., Рыбин В.В. // ФММ. 1976. T. 42. C. 146-154.

[26] Van der Giessen E, Needleman A. // Model Simul. Mater. Sci. Eng. 1995. Vol. 3. P. 689-735.

[27] Aubry S., Rhee M., Hommes G., Bulatov V.V., Arsenlis A. // J. Mech. Phys. Sol. 2016. Vol. 94. P. 105-126.

[28] Kubin L., Devincre B., Hoc T. // Acta Mater. 2008. Vol. 56. N 20. P. 6040-6049.

[29] Arsenlis A., Cai W., Tang M., Rhee M., Oppelstrup T., Hommes G., Pierce1 T.G., Bulatov V.V. // Model. Simul. Mater. Sci. Eng. 2007. Vol. 15. P. 553-595.

[30] Perevezentsev V.N., Sarafanov G.F. // Mater. Sci. Eng. A. 2009. Vol. 503. P. 137-140.

[31] Сарабанов Г.Ф., Перевезенщев В.Н., Свирина Ю.В. // ЖТФ. 2009. Т. 79. Вып. 4. С. 104-110.

[32] Хирт Джс., Лоте И. Теория дислокаций. М.: Атомиздат, 1972. $599 \mathrm{c}$. 